\title{
Article
}

\section{Could knee joint mechanics during the golf swing be contributing to chronic knee injuries in professional golfers?}

Carson, H.J, Richards, James and Coleman, S.G.S

Available at http://clok.uclan.ac.uk/32787/

Carson, H.J, Richards, James ORCID: 0000-0002-4004-3115 and Coleman, S.G.S (2020) Could knee joint mechanics during the golf swing be contributing to chronic knee injuries in professional golfers? Journal of Sports Sciences, 38 (13). pp. 1575-1584. ISSN 0264-0414

It is advisable to refer to the publisher's version if you intend to cite from the work. http://dx.doi.org/10.1080/02640414.2020.1748956

For more information about UCLan's research in this area go to http://www.uclan.ac.uk/researchgroups/ and search for <name of research Group>.

For information about Research generally at UCLan please go to http://www.uclan.ac.uk/research/

All outputs in CLoK are protected by Intellectual Property Rights law, including Copyright law. Copyright, IPR and Moral Rights for the works on this site are retained by the individual authors and/or other copyright owners. Terms and conditions for use of this material are defined in the policies page.

\section{CLoK}

Central Lancashire online Knowledge www.clok.uclan.ac.uk

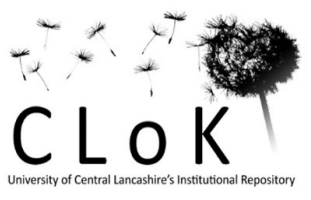


Could knee joint mechanics during the golf swing be contributing to chronic knee injuries in professional golfers?

Key words: golf, driver, kinematics, kinetics, lower limbs

17

*Correspondence concerning this article should be addressed to Howie Carson, 4.19 St

Leonard's Land, Institute for Sport, Physical Education and Health Sciences, Moray House

24 School of Education and Sport, The University of Edinburgh, Edinburgh. EH8 8AQ, United 
27 Full three-dimensional movements and external moments in golfers' knees and the possible involvement in injuries have not been evaluated using motion capture at high sample

frequencies. This study measured joint angles and external moments around the three anatomical axes in both knees of ten professional golfers performing golf drives whilst standing on two force plates in a motion capture laboratory. Significant differences were found in the knee joint moments between the lead and trail limbs for the peak values and throughout all stages during the swing phase. A significantly higher net abduction moment impulse was seen in the trail limb compared with the lead limb $(-0.518$ vs. -0.135 Nms. $\mathrm{kg}^{-1}$ ), indicating greater loading over the whole swing, which could contribute to knee lateral compartment or ACL injuries. A significant correlation $(r=-0.85)$ between clubhead speed at ball contact and maximum joint moment was found, with the largest correlations being found for joint moments at the top of the backswing event and at the end of the follow through. Therefore, although knee moments can contribute to high clubhead speeds, the large moments and impulses suggest that they may also contribute to chronic knee injuries or exacerbate existing conditions. 
The golf swing is a complex sequence of three-dimensional movements with the aim of producing the required clubhead velocities and orientations for a given shot. Key factors to achieve this include the magnitude and timing of muscular forces and moments. Many researchers have studied kinematic and kinetic aspects of the swing since the seminal scientific work of the Golf Society of Great Britain (Cochran \& Stobbs, 1968), with much attention directed towards upper body and trunk/pelvis motion, but little on leg actions during the swing.

This is strange, considering that Cochran and Stobbs stated "make no mistake: the legs and hips are the 'engine' of the swing; the arms and hands are the transmission system" (p. 81; original emphasis). Throughout the swing, the legs are responsible for transferring ground reaction forces and torques to the upper body and onwards to the club. During the backswing, the legs stabilise the pelvis to allow the trunk and shoulders to rotate away from the target, and the magnitude of this rotation has been shown to be positively related to clubhead speed at impact (McLean \& Andrisani, 1997). Geisler (2001) suggested that supination of the front foot and "lateral rotation of the patella" (presumably tibial external rotation) initiate the downswing. After impact the legs are then used to help slow the lower body during the follow through. Knowing the size of the moments and movements within the joints of the lower limbs is therefore very important in helping our understanding of how clubhead velocities are attained. However, currently there have been few studies focussing on leg actions in golf. contribute to injuries (Marshall \& McNair, 2013). A recent systematic review reported that 3$18 \%$ of golfing injuries occurred at the knee, however the reviewed studies gave little information on the exact nature of the injuries or which knee was affected (Baker et al., 2017). 
injuries was comparable to high-impact sports such as basketball. They also identified knee loading as a key factor in establishing knee injury risk mechanisms. Therefore, this aspect of the swing needs further investigation.

Empirically, Gatt, Pavol, Parker and Grabiner (1998) were the first to examine knee kinematics and kinetics during the golf swing and found that in the lead knee, the left knee in right-handed golfers, the peak moments were $20.8 \mathrm{Nm}$ and $96.9 \mathrm{Nm}$ (flexion/extension), $16.1 \mathrm{Nm}$ and 27.7 $\mathrm{Nm}$ (internal/external rotation) and $63.7 \mathrm{Nm}$ and $24.4 \mathrm{Nm}$ (abduction/adduction). The respective values for the trail knee, the right knee in right-handed golfers, were $68.4 \mathrm{Nm}, 58.6$ $\mathrm{Nm}$ (flexion, extension), $19.6 \mathrm{Nm}, 19.1 \mathrm{Nm}$ (internal/external rotations) and $38.8 \mathrm{Nm}, 52.6$ $\mathrm{Nm}$ (abduction/adduction). The authors concluded that while these values were not high enough for golf to be considered an activity with a high risk of traumatic knee injury for healthy individuals, they could be of concern for those rehabilitating after ACL reconstruction or with other knee pathologies. Lynn and Noffal (2010) measured external abduction and adduction moments in the lead knee with the lead foot in a 'square' (neutral) position and with $30^{\circ}$ of external rotation. Mean peak external adduction moments were 0.63 and $0.54 \mathrm{Nm} \cdot \mathrm{kg}^{-1}$, and abduction peak moments were 0.70 and $0.80 \mathrm{Nm} \cdot \mathrm{kg}^{-1}$ for the neutral and the externally rotated foot positions respectively. The authors pointed out that these values were higher than those for gait, stair climbing and drop jump landings but lower than those for side-cutting manoeuvres. They concluded that using an externally rotated lead foot position could possibly slow cartilage wear in healthy individuals and decrease pain in those with medial knee pathology. More recently, Choi, Sim and Mun (2015) studied knee flexion and extension kinetics and kinematics during drives of skilled and unskilled golfers. They found peak extension moments of approximately $0.5-0.7 \mathrm{Nm} \cdot \mathrm{kg}^{-1}$ in the lead leg during the downswing in the skilled golfers but clear extension peaks were not evident in the lead leg data of the 
unskilled group. Although there are no definitive magnitudes for injury-causing moments in golf, the values obtained were higher than those of $0.46 \mathrm{~N} \cdot \mathrm{m} \cdot \mathrm{kg}^{-1}$ for gait (Meireles, De Groote, Van Rossoma, Verschueren, \& Jonkers, 2017).

Thorp et al. (2006) noted that a single peak external moment only reflects the load on a joint at a single time point, however this does not account for the combined load throughout the duration of the movement. During gait, individuals ambulate at different speeds, therefore a variable which incorporates both knee moment and the duration of the movement is needed. Thorp et al. therefore calculated knee adduction angular impulse to enable the understanding of knee loading over the whole stance phase of gait and its relationship to medial OA and found higher values $\left(0.20\right.$ vs. 0.11 N.m.s.kg $\left.{ }^{-1}\right)$ in patients with moderate OA than healthy participants. As the duration of the golf swing is different between individuals, knee adduction/abduction angular impulse could also be valuable to quantify knee loading in golf. This would allow a further exploration of the peak knee abduction moments which were found to be greater than peak adduction moments in golf by Lynn and Noffal (2010). Similarly, Devita, Hunter and Skelly (1992) used extension angular impulses to assess the effects of knee braces on ACL-deficient patients, and so the present study will assess angular impulses in all directions (extension/flexion, adduction/abduction and internal/external rotation).

Notably, there have been a number of methodological issues with previous biomechanics research investigating joint moments during the golf swing. Firstly, several studies have used low sample rates of $60-100 \mathrm{~Hz}$ for kinematic data collection. This, combined with low filter cut-off frequencies, could lead to underestimation of peak values, particularly in the higher derivatives used to calculate kinetic data in a fast action such as the golf swing. Secondly, three studies utilised marker sets which do not allow six degrees of freedom analysis and may cause 
errors in kinematic and kinetic data or miss important axes of motion (Richards, 2018). Thirdly, only one paper allowed participants to use cleated golf shoes, whereas others used golfers in regular athletic shoes or did not state the shoes used. Worsfold, Smith and Dyson (2008) have shown that there are differences in ground reaction torques between cleated and flat-soled shoes and thus this factor could have an important effect on knee moments.

Within the limited number of studies conducted in this area, none have measured threedimensional knee kinematics and kinetics in highly skilled golfers driving the ball when wearing cleated shoes. Therefore, the purpose of this study was to quantify three-dimensional knee joint kinetics and kinematics in the drives of professional golfers and, to examine how external knee moments were related to clubhead speed. Furthermore, the differences in external moments and impulses between lead and trail knees were compared to help identify which limb was more at risk of possible injuries.

\section{Methods}

Participants

Ten right-handed male golfers $\left(M_{\mathrm{age}}=32.0 \pm 9.3\right.$ years, $M_{\mathrm{body}}$ mass $\left.=79.03 \pm 11.12 \mathrm{~kg}\right)$ volunteered to take part. All participants were PGA professionals, which means that they do not have current handicaps, but would have had to have handicaps of $\leq 4$ to gain professional status. The current handicap upper limit for CONGU Category 1 golfers is 5.4 (CONGU, 2018), indicating that the golfers in the present study can be classed as highly skilled. Ethical approval was gained from the University's Ethics Committee, and prior to participation golfers signed a consent form after reading an information sheet. All participants were free from musculoskeletal injuries at the time of testing. 
147 Retro-reflective markers (10 mm diameter) were attached by the same experimenter to each 148 golfer's body. The lower limbs were marked by attaching the markers on right and left sides at 149 the following anatomical landmarks; greater trochanter, medial and lateral femoral condyles, medial and lateral malleoli, $1^{\text {st }}$ and $5^{\text {th }}$ metatarsal heads, calcaneus and the dorsal surface of the foot. Rigid clusters consisting of four markers were also attached to the lateral surfaces of the thigh and shank segments, approximately halfway between their proximal and distal landmarks. Seven retro-reflective markers $(6 \mathrm{~mm})$ were attached to the head of the golf club; four on the clubface and three on the crown (top) of the head. A ball was also marked with retro-reflective tape. A cross of four markers was placed on the ground to aid with alignment and provide reference directions (Figure 1). In addition, a marker was placed on the dorsal surface of the left hand to enable the end of the swing to be identified.

All golfers wore their own golf shoes and shorts. Participants carried out individualised warmups consisting of stretches and practice tee shots. A static calibration trial for $1 \mathrm{~s}$ was collected with the golfer in the anatomical standing position. They then performed eight drives with their own drivers aiming to hit a marked squash ball to a vertical target placed $15 \mathrm{~m}$ away. Any drives which the golfers were unhappy with were repeated. 
Participants performed shots whilst standing on artificial turf, which was attached with twosided tape to the top of two Force Plates (AMTI BP400600, AMTI, USA), ensuring that the golfers had one foot on each plate. Ground reaction force data were sampled at $300 \mathrm{~Hz}$. The retro-reflective markers were tracked using a 10 camera Qualisys Oqus 700 system (Qualisys Medical AB, Sweden) running at $300 \mathrm{~Hz}$, which was synchronised with the force plates. Each corner of both force plates were located in the motion capture coordinate system using reflective markers which were then removed before golf testing. This calibration was repeated before every testing session. The laboratory global coordinate system is shown in Figure 1.

\section{Data processing}

Four swing events were identified: Takeaway (TA; defined as when clubhead linear speed crossed a threshold value of $0.0 \mathrm{~ms}^{-1}$ ); Top of Backswing (TBS; defined when the club linear velocity in the global $z$ direction reached its lowest negative value); Ball Contact (BC; defined as the frame immediately prior to the ball recording a positive linear speed) and Finish (FIN; defined as when the left hand linear velocity in the global $x$-axis crossed a threshold of $0.0 \mathrm{~ms}^{-1}$ after impact). These events were defined in the same way as reported by Carson, Richards and Mazuquin (2019). Three swing phases were delineated by these four events: Backswing (TA to TBS), Downswing (TBS to BC) and Follow through (BC to FIN). This is fewer phases than other studies (e.g., Ball \& Best, 2007), but it has been noted in other activities, such as countermovement jumps, that having more events does not necessarily better predict performance (Moudy, Richter \& Strike, 2018). Therefore, three phases were chosen for simplicity and relevance for golf coaches and players. 
Due to problems in viewing markers, not all trials were successfully tracked for all golfers. At least five trials were available for each golfer, so raw kinematic and kinetic data for all successfully-tracked trials (i.e., between five and eight) per participant were exported as c3d files into Visual 3D v6.01.03 software (C-Motion Inc., USA). Kinematic and force plate data were filtered using Generalised Cross Validated Quintic Splines (Woltring, 1985), which has been shown to be a valid and objective method of smoothing sporting movement (Challis \& Kerwin, 1988, Giakas \& Baltzopoulos, 1997). Knee joint angles were calculated using an XY-Z Cardan sequence (flexion/extension $[\mathrm{X}]$, abduction/adduction [Y], internal/external rotation [Z]). External knee moments were also calculated in Visual 3D with the shank as the reference segment and were normalised to the participant's body mass (Lynn \& Noffal, 2010; Baker et al., 2017). Positive joint angles around the $\mathrm{X}, \mathrm{Y}$ and $\mathrm{Z}$ axes represented flexion, abduction and external rotation of both knees. Positive moments around $\mathrm{X}, \mathrm{Y}$ and $\mathrm{Z}$ were extension, adduction and internal rotation for both knees (Lynn \& Noffal, 2010). External knee angular moment impulses were calculated by the separate integration of the positive and impulses.

Kinematic and kinetic data were time-shifted so that $\mathrm{BC}$ was coincident at time $=0.0 \mathrm{~s}$ for all golfers. Data were not normalised or event warped, as these manipulations affect higher derivatives and often obscure the clarity of time series graphs. Peak knee moments around each axis were identified from the data, including which phase they were in, and moments at the four swing events were also identified. 
Knee moments at the four swing events (TA, TBS, BC, FIN) and maximum and minimum values were compared between the lead and trail limbs. Data were checked for normality with Shapiro-Wilk tests with an $\alpha$-level of 0.05 , and if found to be normally distributed, left and right data were compared using dependent $t$-tests with a Bonferroni-adjusted $\alpha$-level of 0.003 (calculated as $0.05 / 18$ tests). If data were found to be not normally distributed a Wilcoxon Matched Pairs Signed Ranks test was carried out. Effect sizes were classified by Cohen's $d$ (Cohen, 1992) and 95\% confidence limits were calculated for each comparison.

Knee angular impulses for the lead and trail legs were tested for normality and then compared using dependent $t$-tests with a Bonferroni-adjusted $\alpha$-level of $0.006(0.05 / 9$ tests $)$ or a Wilcoxon Matched Pairs Signed Ranks if not normally distributed, and effect sizes were classified by Cohen's $d$ (Cohen, 1992).

Clubhead speed at BC was correlated with knee joint moments at TBS, BC, FIN and peak values using Pearson Product Moment Correlations with a Bonferroni-adjusted $\alpha$-level of 0.003. For data that was not normally distributed a Spearman Rank Order correlation was carried out. Correlation effect sizes were categorised by the reference values for correlations (0.1 small; 0.3 moderate; 0.5 large; 0.7 very large; 0.9 nearly perfect) given by Hopkins, Marshall, Batterham and Funin (2009).

\section{Results}

The mean $( \pm \mathrm{SD})$ duration of the three phases (Backswing, Downswing and Follow through) were $0.864 \pm 0.134 \mathrm{~s}, 0.265 \pm 0.043 \mathrm{~s}$ and $0.433 \pm 0.044 \mathrm{~s}$ respectively. Intra-individual variation in phase durations was lower than that between participants, particularly in the 
downswing where each golfer was very consistent with a mean within-participant coefficient of variation of only $2.2 \%$. The mean clubhead speeds at BC were $42.09 \pm 3.15 \mathrm{~m} . \mathrm{s}^{-1}$ with a range of $34.8-47.1 \mathrm{~m} \cdot \mathrm{s}^{-1}$.

Figures $2 a-2 c$ show the three-dimensional knee joint angles for the lead and trail limbs. The solid vertical line crossing the abscissa at time $=0.0$ represents $\mathrm{BC}$ synchronised for all participants and the dotted vertical line represents the mean value for all golfers' TBS. During the backswing, participants displayed knee flexion, adduction and external rotation in the lead limb, with slight knee extension flexion, abduction and internal rotation in the trail limb. Maximal excursions for knee abduction/adduction for the lead limb and external/internal rotation for both limbs were reached at the end of the backswing (TBS). For the first half of the downswing both knees continued to flex but then extended rapidly, with the knee of the lead limb commencing extension just prior to that of the trail limb, although considerable interindividual variations in timing were seen. The knee of the trail limb also adducted slightly in the first part of the downswing followed by slight abduction. The knee of the lead limb abducted rapidly from TBS to BC after which it stayed at a fairly constant angle. The knee on the lead limb internally rotated rapidly from TBS to BC, which was accompanied by knee external rotation in the trail limb.

Figures $3 a-3 c$ show that during the backswing, the knee on the lead limb experienced a flexion moment whilst the knee on the trail limb showed an extension moment. These increased to their peak values approximately halfway through the downswing, after which they decreased to close to zero at BC. During the follow through a small extension moment was seen in the 
knee on the lead limb, which was accompanied by a large knee flexion moment in the trail limb. In the frontal plane, initially both knees experienced small knee abduction moments which increased in the lead limb but decreased in the trail limb during the backswing. At TBS the knee abduction moments increased on both the trail and lead limbs, but the latter then rapidly changed to an adduction moment at BC. During follow through, the lead limb still experienced a knee adduction moment, whereas the trail limb had a slowly decreasing knee abduction moment. During the backswing, the lead limb experienced a knee external rotation moment whereas the trail limb experienced a knee internal rotation moment. After TBS, both knees experienced an external rotation moment, but whilst this was maintained until BC for the trail limb, the lead limb changed to a small internal rotation moment at BC. After impact, the lead limb continued to experience a knee internal rotation moment, with the trail limb showing a slowly decreasing knee external rotation moment. Similar to the movement timing, there were clear inter-individual differences in joint moments during the whole swing, as exemplified by two participants in Figure 4.

Table 1 shows the peak knee joint moments in each anatomical direction (extension/flexion, adduction/abduction and internal/external rotation).

Differences in knee joint moments between lead and trail limbs at swing events and maximum and minimum were all normally distributed apart from peak flexion. Therefore, a Wilcoxon 
carried out for all other contrasts. Results from the statistical tests are in Table 2, these show that ten lead versus trail limb knee moment differences were significant $(p<0.003)$. Of the significant results, seven showed greater knee moments in the lead limb and three showed greater knee moments in the trail limb.

$* * *$ Table 2 here***

External knee angular impulses are shown in Table 3. Statistical comparisons showed that adduction and internal rotation impulses were significantly higher in the lead than in the trail knee with large effect sizes. The abduction magnitude (in the negative direction) was significantly higher in the trail than in the lead knee, again with large effect size. There was a net abduction impulse over the whole swing for both knees, with the trail leg being significantly greater (in negative direction) than the lead leg. There was also an overall net external rotation impulse for both knees, with the lead knee being significantly greater (in the negative direction) than the trail knee.

$* * *$ Table 3 here $* * *$

Correlations between clubhead speed at BC and knee joint moments at TBS, BC and FIN did not produce any significant results: however large-very large effects sizes were found for the relationships between clubhead speed and lead limb knee adduction/abduction moment at TBS $(r=-0.68)$, the lead limb knee internal/external rotation moment at TBS $(r=-0.69)$, and the trail limb knee internal/external rotation moment at FIN $(r=-0.68)$. Correlations of peak joint moments with clubhead speed at BC produced only one significant relationship; with lead limb knee adduction/abduction peak moment $(r=-0.85 ; p=0.002$; effect size very large-near 
perfect), although lead limb knee extension/flexion peak moment showed a large-very large effect size $(r=-0.67)$.

\section{Discussion}

The authors believe this is the first paper to present three-dimensional knee joint kinematics and kinetics in the full swings of professional golfers using six degrees of freedom methods with motion capture at a high sample frequency. The utilisation of golfers' own drivers and golf shoes also meant that this study had greater ecological validity than previous studies.

Knee flexion and extension kinematics of the lead and trail limbs in the swing were very similar to those presented by Choi et al. (2015), but were larger than those presented in other studies (Gatt et al., 1998; Somjarod, Tanawat \& Weerawat, 2011). In the frontal plane, the present study showed knee abduction in the lead limb during the downswing with the trail limb showing slight knee adduction. Although the ranges of motion were comparable to those reported by Gatt et al., there were consistent 'offsets' from their results. Finally, the knee joints showed less external/internal rotation during the downswing than the values presented by Gatt et al. but more than in the paper of Somjarod et al. Although the kinematic curves over the whole swing were similar to the aforementioned studies, differences between the present study and previous research was possibly due to the marker sets and models used. In addition, there were considerable inter-individual differences in the motions of our golfers, a fact also noted by Choi et al., and so individual consideration must be paramount when attempting to translate these data to the applied setting (Ball \& Best, 2012). 
342 Sagittal plane external knee joint moments for the first half of the downswing showed flexion 343 for the lead limb and extension for the trail limb. The peak values shown in Table 1 were slightly above those of Choi et al. (2015) who gave graphical results of approximately -1.00 $\mathrm{Nm} \cdot \mathrm{kg}^{-1}$ and $0.75 \mathrm{Nm} \cdot \mathrm{kg}^{-1}$ respectively, and very similar to those of Gatt et al. $\left(-1.26 \mathrm{Nm} \cdot \mathrm{kg}^{-1}\right.$ and $0.76 \mathrm{Nm} \cdot \mathrm{kg}^{-1}$ ). During the second half of the downswing knee moments were reversed so that at $\mathrm{BC}$ there was a slight knee extension moment for both limbs. In the follow through the lead limb experienced a small knee extension moment, whereas in the trail limb a large knee flexion moment was seen $\left(-0.77 \mathrm{Nm} \cdot \mathrm{kg}^{-1}\right)$.

There has been previous interest in frontal plane knee moments, as it has been suggested that these might lead to acute or chronic knee injuries such as Anterior Cruciate Ligament (ACL) damage and OA. The present study found very similar peak values in the lead limb to the results of Lynn and Noffal (2010). Peak values for adduction moments $\left(M=0.49\right.$ N.m.kg $\left.{ }^{-1}\right)$ were above those reported by Mareiles et al. (2017) for healthy and early OA patients $\left(0.46 \mathrm{Nm} \cdot \mathrm{kg}^{-1}\right)$ but not as high as those with established OA $\left(0.57 \mathrm{Nm}_{\mathrm{kg}}{ }^{-1}\right)$. Interestingly, the present study showed that the trail limb experiences higher knee abduction and lower adduction peak moments than that of the lead limb. The large abduction moment took place just prior to BC (Figure 3) and, whilst the ground reaction forces on the trail limb were small at this time, their direction produced a large moment arm resulting in a large abduction moment. Large abduction moments can lead to ACL stress (Fukuda, Woo \& Loh, 2003) and although this was commented upon by Lynn and Noffal for the lead limb, the greater external abduction moment in the trail limb appears to show a greater risk of ACL injury. This could also be exacerbated by the extension moment present in the trail limb during the downswing. The abduction moment magnitudes were much higher $\left(0.78 \mathrm{Nm} \cdot \mathrm{kg}^{-1}\right.$ and $0.87 \mathrm{Nm} \cdot \mathrm{kg}^{-1}$ in the lead and trail knee respectively) than those in adduction, and well above those reported for established OA 
in adduction. The possible injury risks associated with external abduction moments were reinforced by the abduction moment impulses for both knees over the whole swing, with the trail limb again showing higher values. High impulses $\left(>0.20\right.$ N.m.s.kg $\left.{ }^{-1}\right)$ due to adduction have been shown to be linked to medial OA (Thorp et al., 2006), so the much higher abduction magnitudes (0.34 N.m.s. $\mathrm{kg}^{-1}$ for lead and 0.55 N.m.s.kg ${ }^{-1}$ for trail knees) in this study may be linked to lateral compartment problems. Although lateral OA is much less common than medial OA, with $10 \%$ lateral compartment versus $90 \%$ medial compartment (Scott, Nutton \& Biant, 2013), there is little information available on the prevalence of these conditions in golfers. This confirms the findings of Mündermann, Dyrby, D’Lima, Colwell and Andriacchi (2008), who used an instrumented total knee replacement and found that the golf swing had $40 \%$ more loading on the lateral compartment compared to the medial. Future research should aim to assess moment values in golfers suffering from knee pain to better illuminate our understanding and provide meaningful indicators of risk.

The large-very large effect sizes for the relationships between clubhead speed at BC and the knee abduction moment and external rotation moment on the lead limb at TBS can be linked to the need to stabilise the pelvis in the backswing in order to generate a maximal differential in shoulder-hip rotation, sometimes called the "X-Factor" (McLean \& Andrisani, 1997). This 
is also supported by the significant correlation between lead knee peak abduction moment (at $\sim 40 \%$ of the downswing) and clubhead speed at BC. The large-very large effect size for the correlation between the knee external rotation moment in the trail limb at FIN and clubhead speed at BC may relate to the moments needed to slow the clubhead and to maintain balance at FIN.

There were several limitations of this research. Firstly, the use of a squash ball instead of a golf ball was chosen due to safety reasons in the laboratory. Impact characteristics between the club head and a squash ball are different to those with a golf ball and due to the smaller mass of the squash ball the club head will have decelerated less at impact. This might have changed swing biomechanics during the Follow through and thus joint moments at FIN may have been different than if a golf ball had been used. Nevertheless, joint moments at the other swing events are unlikely to be different because the golfers, when asked after the testing sessions, all reported that they had performed their normal swings. Another limitation was the small homogenous sample size affecting statistical power and possibly obscuring theoretical correlations. However there was large variation in some of the dependent variables (e.g., joint moments; Figure 4), showing that even between participants with similar characteristics there may be important individual differences. This means that each golfer needs individual analysis to ascertain key factors such as knee abduction moments and moment impulse, as injury risks may be different with different swings. This has already been pointed out in other aspects of golf research (Ball \& Best, 2012) but also applies to knee kinetics and kinematics. It may also mean that more sophisticated analysis techniques, such as Statistical Parametric Mapping may reveal more than the differences found in the present study.

\section{Conclusions}


417 This study showed that golfers undergo knee joint external moments during the golf swing 418 which, while are not usually of sufficient magnitude to directly cause acute injuries, may 419 contribute to chronic knee injuries or be hazardous to those with pre-existing conditions. 420 Whereas previous studies have concentrated on the lead limb, this paper showed that the trail 421 limb also experiences influential moments and associated loads on key structures. The large 422 abduction moments and impulses suggest that load is placed particularly on the lateral 423 compartment of the knee and might also stress the ACL. The large-very large effect sizes for 424 correlations between external knee moments, particularly at TBS and early downswing, and 425 the significant correlation between lead knee abduction moment with clubhead speed at BC, 426 support the statement of Cochran and Stobbs (1968) that the legs are "the engine of the swing". 


\section{References}

428

Baker, M.L., Epari, D.R., Lorenzetti, S., Sayers, M., Boutellier, U., \& Taylor, W.R. (2017). Risk factors for knee injury in golf: A systematic review. Sports Medicine, 47, 26212639. doi:10.1007/s40279-017-0780-5

Ball, K.A., \& Best, R.J. (2007). Different centre of pressure patterns within the golf stroke I: Cluster analysis. Journal of Sports Sciences, 25, 757-770. doi:10.1080/02640410600874971

Ball, K., \& Best, R. (2012). Centre of pressure patterns in the golf swing: Individual-based analysis. Sports Biomechanics, 11, 175-189. doi:10.1080/14763141.2012.673007

Carson, H.J., Richards, J., \& Mazuquin, B. (2019). Examining the influence of grip type on wrist and club head kinematics during the golf swing: Benefits of a local co-ordinate system European Journal of Sport Science, 19, 327-335. doi:10.1080/17461391.2018.1508504

Challis, J. H., \& Kerwin, D.G. (1988). An evaluation of splines in biomechanical data analysis. In Biomechanics XI-B (pp. 1057-1061). Basel: Karger.

Choi A., Sim T., \& Mun J.H. (2015). Quasi-stiffness of the knee joint in flexion and extension during the golf swing. Journal of Sports Sciences, 33, 1682-1691. doi:10.1080/02640414.2014.1003591

Cochran, A, \& Stobbs, J. (1968). The search for the perfect swing. Philadelphia: J. Lippincott Co.

Cohen, J. (1992). A power primer. Psychological Bulletin, 112, 155-159. doi:10.1037/00332909.112.1.155

CONGU. (2018). A quick guide to the CONGU® handicapping system: For players. Retrieved from $\quad$ http://www.congu.co.uk/wp-content/uploads/2017/12/Players-quick-guide2018.pdf 
Devita, P., Hunter, P.B., \& Skelly, W.A. (1992). Effects of a functional knee brace on the biomechanics of running. Medicine and Science in Sports and Exercise, 24, 797-806.

Fukuda, Y., Woo, S.L.-Y., Loh, J.C., Tsuda, E., Tang, P., McMahon, P.J. \& Debski, R.E. (2003). A quantitative analysis of valgus torque on the ACL: A human cadaveric study. Journal of Orthopaedic Research, 21, 1107-1112. doi:10.1016/S0736-0266(03)000846

Gatt Jr., C.J., Pavol, M.J., Parker, R.D., \& Grabiner, M.D. (1998). Three-dimensional knee joint kinetics during a golf swing. Influences of skill level and footwear. American Journal of Sports Medicine, 26, 285-294. doi:10.1177/03635465980260022101

Geisler, P.R. (2001). Golf. In E. Shamus \& J. Shamus (Eds.), Sports injury prevention and rehabilitation (pp. 185-226). New York: McGraw-Hill.

Giakis, G., \& Baltzopoulos, V. (1997). A comparison of automated filtering techniques applied to biomechanical walking data. Journal of Biomechanics, 30, 747-850. doi:10.1016/S0021-9290(97)00042-0

Hopkins, W.G., Marshall, S.W., Batterham, A.M., \& Hanin, J. (2009) Progressive statistics for studies in sports medicine and exercise science. Medicine and Science in Sports and Exercise, 43, 3-10. doi:10.1249/MSS.0b013e31818cb278

Lynn, S.K., \& Noffal, G.J. (2010). Frontal plane knee moments in golf: Effect of target side foot position at address. Journal of Sports Science and Medicine, 9, 275-281.

Marshall, R.N., \& McNair, P.J. (2013). Biomechanical risk factors and mechanisms of knee injury in golfers. Sports Biomechanics, 12, 221-230 doi:10.1080/14763141.2013.767371

McLean, J., \& Andrisani, J. (1997). The X-factor swing. New York: Harper Collins.

Meireles, S., De Groote, F., Van Rossoma, S., Verschueren, S., \& Jonkers, I. (2017) Differences in knee adduction moment between healthy subjects and patients with 
osteoarthritis depend on the knee axis definition. Gait \& Posture, 53, 104-109. doi.10.1016/j.gaitpost.2017.01.013

Meyer, E.G., \& Haut, R.C. (2008). Anterior ligament injury induced by internal tibial torsion or tibiofemoral compression. Journal of Biomechanics, 41, 3377-3383. doi:10.1016/j.jbiomech.2008.09.023

Moudy, S., Richter, C., \& Strike, S. (2018). Landmark registering waveform data improves the ability to predict performance measures. Journal of Biomechanics, 78, 109-117. doi: 10.1016/j.jbiomech.2018.07.027

Mündermann, A., Dyrby, C.O., D’Lima, D.D., Colwell, C.W., \& Andriacchi, T.P. (2008). In vivo loading characteristics during activities of daily living as measured by an instrumented total knee replacement. Journal of Orthopaedic Research, 26, 11671172. doi:10.1002/jor.20655

Richards, J. (Ed.). (2018). The comprehensive textbook of clinical biomechanics (2 ${ }^{\text {nd }}$ edition). Elsevier Health Sciences.

Scott, C.E.H., Nutton, R.W., \& Biant, L.C. (2013). Lateral compartment osteoarthritis of the knee. The Bone \& Joint Journal, 95, 436-444. doi:10.1302/0301-620X.95B4.30536

Somjarod, M., Tanawat, V., \& Weerawat, I. (2011). The analysis of knee joint movement during golf swing in professional and amateur golfers. World Academy of Science, Engineering and Technology, International Journal of Social, Behavioral, Educational, Economic, Business and Industrial Engineering, 5, 545-548.

Thorp, L.E., Sumner, D.R., Block, J.A., Moisio, K.C., Shott, S., \& Wimmer, M.A. (2006). Knee joint loading differs in individuals with mild compared with moderate medial knee osteoarthritis. Arthritis \& Rheumatism, 54, 3842-3849. doi:10.1002/art.22247 
500 Woltring, H.J., (1985). On optimal smoothing and derivative estimation from noisy displacement data in biomechanics. Human Movement Science, 4, 229-245. doi:10.1016/0167-9457(85)90004-1

503 Worsfold, P., Smith, N.A., \& Dyson, R.J. (2008). Low handicap golfers generate more torque at the shoe-natural grass interface when using a driver. Journal of Sport Science and Medicine, 7, 408-414. 


\section{Figure Captions}

Figure 1. Marker sets on lower limbs, golf clubhead and ground reference with lab coordinate system.

Figure 2. Flexion/extension (a), abduction/adduction (b) and external/internal rotation (c) angles of the lead (left) and trail (right) knee joints during the swing.

Figure 3. Extension/flexion (a), adduction/abduction (b) and internal/external (c) joint moments of the lead (left) and trail (right) knee joints during the swing.

Figure 4. Exemplars of inter-individual differences in knee moments and timing across extension/flexion (a), adduction/abduction (b) and internal/external (c). 